\title{
Le modèle du soi peptidique
}

Le « soi », du point de vue du système immunitaire, serait défini par un ensemble de peptides dérivés des protéines cellulaires et présentés aux lymphocytes $T$ dans le contexte des molécules HLA chez l'homme (ou H2 chez la souris). Cette proposition générale, qui fonde le modèle du «soi peptidique», conduit à distinguer un «soi somatique», représenté par des peptides dérivés des protéines générales, et un «soi immunologique» dont le support serait des «idiopeptides » (peptides provenant des régions variables des anticorps et des récepteurs pour l'antigène des cellules $T$ ). Les lymphocytes $T$ seraient tolérants au « soi somatique » et éventuellement réactifs au «soi immunologique », ce qui pourrait être à la base d'un réseau idiopeptidique. La théorie du «soi peptidique» permet d'interpréter certains phénomènes d'auto-immunité et d'envisager des manipulations de la réponse immune ouvrant d'importantes perspectives dans le domaine des vaccinations pratiques.

\section{Philippe Kourilsky}

Directeur de recherche au Cnrs. Directeur de l'unité 277 de l'Inserm

\section{Jean-Michel Claverie}

Chef de laboratoire à l'Institut Pasteur. Chargé de recherche au Cnrs

\footnotetext{
ADRESSE

Ph. Kourilsky, J.-M. Claverie : unité de biologie moléculaire du gène, Inserm U. 277, et unité d'informatique scientifique, Institut Pasteur, 25, rue du Docteur-Roux, 75724 Paris Cedex 15, France.
}

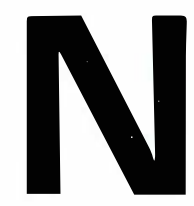

ous avons élaboré le modèle du « soi peptidique » en $1986[1]$ et, lui trouvant un pouvoir explicatif surprenant, en avons explicité les conséquences logiques par la suite [2, 3]. L'ensemble s'inscrit bien entendu dans un mouvement très vivace de l'immunologie expérimentale et théorique, et certaines idées semblables, exprimées par d'autres, recoupent divers segments du modèle [4-9]. Ce dernier reste, à ce jour, largement spéculatif et doit être considéré comme une source d'hypothèses de travail qui se trouveront validées ou invalidées par l'expérience.

\section{Présentation de peptides par les antigènes du CMH}

Une série de travaux remarquables menés par quelques groupes au cours des quinze dernières années a conduit à deux notions essentielles: (a) les antigènes «étrangers (qui suscitent une réponse immunitaire de type humoral ou cellulaire) subissent en général une dégradation intracellulaire, de sorte que ce sont des fragments d'antigènes (des peptides) qui sont reconnus par les cellules T; (b) la fonction des antigènes du complexe majeur d'histocompatibilité (CMH) est donc la suivante : ce sont, en fait, des récepteurs de peptides dotés d'une certaine spécificité, de telle sorte que différents antigènes du CMH (c'est-à-dire différents allèles dans divers haplotypes, ou différents antigènes dans le même haplotype) lieront (c'est-à-dire présenteront) mieux certains peptides que d'autres.

Ces notions reposent avant tout sur un travail considérable effectué sur la fonction des antigènes de classe II. Les travaux effectués plus récemment avec les antigènes de classe I par Townsend $e t$ al. $[10,11]$ puis par Maryanski et al. [12] montrent que ceux-ci sont aussi des «présentoirs de peptides ». La structure tridimensionnelle de HLA-A2, récemment élucidée [13, 14] est, à cet égard, particulièrement éclairante. Elle révèle deux hélices $\alpha$, posées sur un plateau de feuillets $\beta$, l'ensemble constituant très probablement la «niche » à peptides. La structure des antigènes de classe II est probablement assez semblable 


\section{REFEERENCES}

1. Kourilsky P, Claverie JM. (1986) The peptidic self model : a hypothesis on the molecular nature of the immunological self. $A n n$ Immunol Inst Pasteur (Paris) 1986 ; 137 D : 321.

2. Claverie JM, Kourilsky P. The peptidic self model : a reassessment of the role of the major histocompatibility complex molecules in the restriction of the T-cell response. Ann Immunol Inst Pasteur (Paris) 1986; 137 D : 425-42.

3. Kourilsky P, Chaouat G, RabourdinCombe C, Claverie JM. Working principles in the immune system implied by the «peptidic self » model. Proc Natl Acad Sci USA 1987 ; 84 : 3400-4.

4. Grabar P. « Self $\star$ and « not-self $\star$ in immunology. Lancet 1974; I : 1320-2.

5. Benacerraf B. A hypothesis to relate the specificity of $T$ lymphocytes and the activity of I region-specific Ir genes in macrophages and B lymphocytes J Immunol 1978; 120 . (6) : 1809-12

6. Winchester G, Sunshine $\mathbf{G H}$, Nardi $\mathbf{N}$ Mitchison NA. Antigen-presenting cells do not discriminate between self and nonself. Immunogenetics $1984 ; 19$ : 487-91.

7. Jorgensen $T$, Bogen B, Hannestad $K$ Recognition of variable (V) domaine of mye loma protein 315 by B- and 5-lymphocytes. In : Janeway C, Sercarz E, Wigzell H, eds Immunoglobulin Idiotypes. ICN-UCLA Sym posia on Molecular and Cellular Biology, vol. XX. New York, Londres, Academic Press : 1981, 573.

8. Leserman L. The introversion of the immune response : a hypothesis for $\mathrm{T}-\mathrm{B}$ reaction. Immunol Today 1985 ; 6 : 352-5.

9. Werdelin $O$. T cell recognize antigen alone and not MHC molecules. Immunol Today $1987 ; 8: 80-4$

10. Townsend ARM, Gotch FM, Davey Y. Cytotoxic $\mathrm{T}$ cells recognize fragments of influenza nucleoprotein. Cell 1985; 42 : 457 . 67.

11. Townsend ARM, Rothbard J, Gotch FM, Bahadur G, Wraith D, McMichael AJ. The epitopes of influenza nucleoprotein recognized by cytotoxix $T$ lymphocytes can be defined with short synthetic peptides. Cell 1986 ; 44 : 959-68.

12. Maryanski JM, Pala P, Corradin G, Jordan BR, Cerottini JC. $\mathrm{H}-2$ restricted cytolytic $T$ cells specific for HLA can recognize a synthetic HLA peptide. Nature 1986; 324 : 578-9.

13. Bjorkman PJ, Saper MA, Samraoui B, Bennett WS, Strominger JL, Wiley DC. Structure of the human class I histocompatibility antigen, HLA-A2. The foreign antigen binding site and $T$ cell recognition regions of class I histocompatibility antigens. Nature 1987 ; 329 : $506-18$.
[13]. Il convient de souligner que, si des peptides synthétiques exogènes s'avèrent fonctionnels dans la liaison aux antigènes du $\mathrm{CMH}$ et/ou dans les tests de présentation, la structure moléculaire exacte des fragments d'antigène présentés est inconnue. Dans certains cas, il peut arriver qu'il y ait présentation de l'antigène non fragmenté. En résumé, qualifier les antigènes du $\mathrm{CMH}$ de « récepteurs de peptides» constitue un raccourci de langage utile, qui ne doit pas occulter une réalité plus complexe.

De très nombreuses expériences, effectuées d'abord avec les antigènes du CMH de classe II, et plus récemment avec les antigènes de classe I, montrent que ces récepteurs de peptides sont dotés d'une certaine spécificité. Ainsi, la plupart des peptides essayés à ce jour, qui se lient à un antigène du $\mathrm{CMH}$ déterminé (ou entrent en compétition avec un peptide qui se lie) ne s'attachent pas à d'autres antigènes du CMH qui ont été testés [15]. Les données actuelles ne permettent pas encore d'apprécier toute la rigueur de la spécificité de cette association. Il est néanmoins frappant de constater que la structure tridimensionnelle de HLA-A2 place la majorité des résidus dits «polymorphes» (c'est-à-dire variables dans les antigènes de divers haplotypes) dans la poche à peptides [13]. De même, les mutations de $\mathrm{K}^{\mathrm{b}}$ chez la souris (mutants $\mathrm{K}^{\mathrm{bml}}$, etc.) altèrent des acides aminés pour la plupart situés dans ce site [13, 14]. On peut ainsi comprendre qu'une altération, même d'un seul résidu, puisse modifier le répertoire des peptides présentés et ainsi altérer profondément la réponse immunitaire.

\section{Épitopes B et épitopes $T$}

La nature radicalement différente des épitopes $T$ (fragment d'antigènes presenté par les molécules du $\mathrm{CMH}$ au récepteur des cellules $T$ ) et des épitopes $B$ (région de l'antigène reconnue par un anticorps) a de nombreuses et pro- fondes conséquences [3]. La première touche à la coopération entre cellules B et $T$ qui peut faire intervenir non pas un pont antigénique comme on l'a cru pendant longtemps, mais un pont peptidique (figure 1). Les cellules B semblent en effet jouer un rôle de cellules présentatrices plus général qu'on ne l'avait supposé. L'antigène étranger, complexé par l'immunoglobuline spécifique présente à la surface de la cellule B, est internalisé et représenté sous forme de peptides [16, 17]. Dans ce processus, l'antigène étranger se trouve concentré dans les cellules B de spécificité appropriée.

L'épitope reconnu par le lymphocyte $\mathrm{T}$ (en somme responsable d'un «bon à tirer » de l'anticorps) peut n'avoir aucune parenté moléculaire avec la cible initiale de l'anticorps. Ainsi, un lymphocyte $B$ synthétisant un anticorps contre la protéine d'enveloppe d'un virus, mais présentant des peptides issus de toutes les protéines virales, peut se voir stimulé par un lymphocyte $T$ spécifique d'un peptide (épitope $T$ ) issu d'une nucléoprotéine interne. Un tel phénomène a déjà été observé dans le cas du virus de l'hépatite B [19]. Cette nouvelle conception de la collaboration T-B permet d'envisager des manipulations nouvelles et quelquefois paradoxales de la réponse immunitaire, en particulier pour des antigènes complexes (virus, bactéries). La figure I suggère également un mécanisme simple pour l'apparition d'auto-anticorps après rupture de la tolérance par des antigènes ressemblant à des protéines du soi. Deux protéines, l'une étrangère, l'autre du soi, mais portant un épitope B commun, sont susceptibles d'internalisation et de présentation par le même lymphocyte $B$. La présentation d'un épitope $T$ n'appartenant pas au soi peptidique à un lymphocyte $T$ auxiliaire déclenchera la synthèse d'anticorps antisoi [3].

$\mathrm{La}$ manipulation des réponses immunitaires avec des antigènes couplés répond aussi, en première 
Pont antigénique

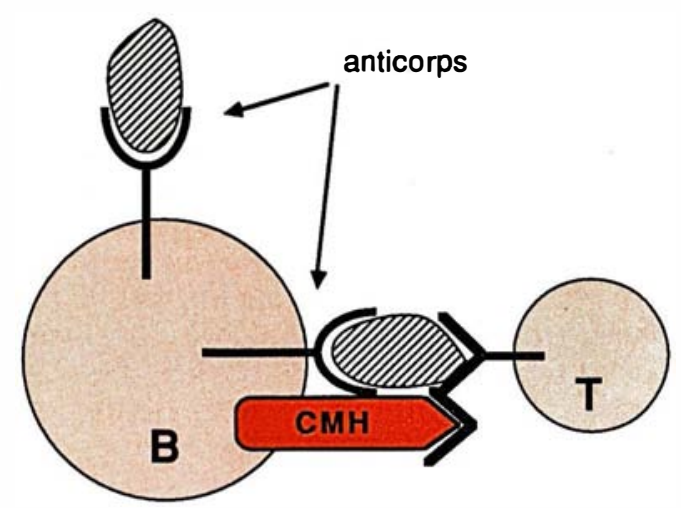

Pont peptidique

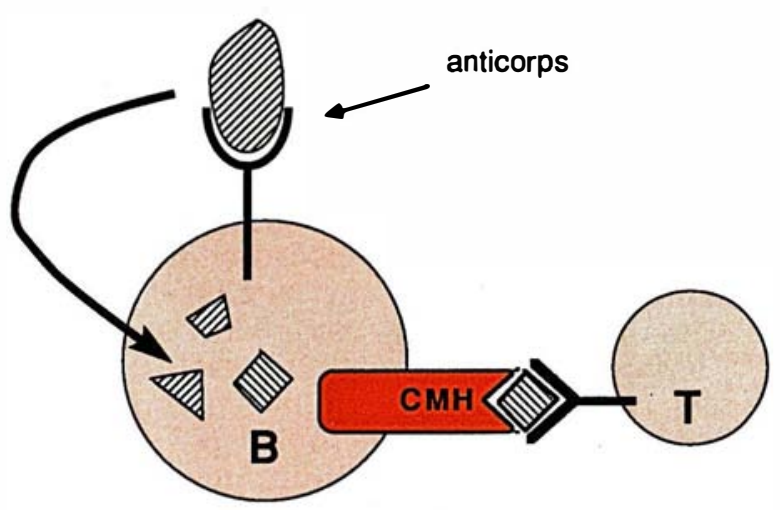

lors que cette dernière fournit les épitopes $T$ nécessaires à l'activation de la réponse. Des peptides synthétiques pourraient aussi être utilisés comme épitopes $T$ qui serviraient à régler la réponse immunitaire. Ces notions pourraient être d'une grande importance dans l'élaboration d'une nouvelle génération de vaccins synthétiques. Deux facteurs limitants devront néanmoins être pris en compte: le premier est la restriction HLA. En effet, dans la mesure où les antigènes du $\mathrm{CMH}$ sont sélectifs vis-à-vis des peptides qu'ils présentent, des épitopes $T$ synthétiques pourraient n'être actifs que dans un nombre restreint d'haplotypes du CMH. Ce problème peut être résolu par l'emploi d'un mélange d'épitopes $T$ ou d'une protéine porteuse immunogène génératrice d'épitopes $\mathrm{T}$. Une idée consiste à utiliser comme protéines porteuses des protéines qui ont déjà fait leurs preuves dans des vaccinations (par exemple, la toxine tétanique). Ici intervient le phéno$\mathrm{m}$ è $\mathrm{n} e \mathrm{~d} \mathrm{e} \ll \mathrm{s} u \mathrm{p} \mathrm{p}$ e s s ion épitopique »: la réponse contre un peptide couplé à une protéine porteuse est en général bien moindre si l'organisme a déjà été immunisé contre cette protéine porteuse $[3,18]$.

\section{Le soi peptidique}

Figure 1. La collaboration entre lymphocytes B et lymphocytes $T$. On a longtemps cru que la collaboration entre les lymphocytes $B$ (qui synthétisent les anticorps) et les lymphocytes $T$ (qui « orchestrent » la réponse immune) imposait l'établissement d'un pont antigénique, le récepteur du lymphocyte T reconnaissant l'antigène présenté «dans le contexte» des antigènes $\mathrm{CMH}$ de classe II. Des expériences récentes [16, 17] intégrées dans le modèle du soi peptidique [1] suggèrent que l'antigène effectivement reconnu par le récepteur du lymphocyte $T$ est un " sous-produit » (un peptide) de l'antigène capté par l'anticorps internalisé et dégradé. Ce peptide est sélectionné par l'antigène CMH qui en assure la présentation à la surface. La collaboration entre lymphocyte $B$ et $T$ met alors en œuvre un pont peptidique. L'interaction des lymphocytes $T$ et des macrophages (ou des cellules dendritiques, ou toutes autres cellules présentatrices) obéit à un schéma similaire. $\mathrm{CMH}=$ complexe majeur d'histocompatibilité.

approximation, à une logique simple qui rend assez bien compte des immunisations obtenues avec des peptides. Il n'y a aucune raison, en effet, qu'un épitope $B$ soit aussi un épitope $T$. Si les épitopes B sont localisés à la surface des molécules d'antigènes et, à ce titre, souvent correc$m / s n^{\circ} 3$ vol. 4, mars 88 tement identifiés par des algorithmes utilisant des paramètres tels que l'accessibilité au solvant, il n'y a pas la moindre raison que ces derniers prédisent convenablement les épitopes T. Le couplage d'un épitope B (sous forme d'un peptide synthétique) avec une protéine porteuse est efficace dès

Le modèle du soi peptidique intègre les notions précédentes et les organise autour de l'hypothèse unificatrice fondamentale que des peptides dérivés des protéines de l'organisme (y compris les protéines intracellulaires) peuvent être présentés à la surface des cellules par les antigènes du $\mathrm{CMH}$ de classe I et de classe II. Cette hypothèse constitue une généralisation logique des expériences de Townsend et al. [10, 11] sur la reconnaissance de la nucléoprotéine de la grippe par des cellules T cytotoxiques (CTL). Des fibroblastes «naïfs » transfectés par le gène viral codant pour cette protéine nucléaire sont des cibles pour certains clones de CTL, dont il est montré par ailleurs qu'ils 


\section{RÉFÉRENCES}

14. Kahn A. La troisième dimension pour les molécules de classe I du complexe majeur d'histocompatibilité : un cristal de HLA-A2 médecine/science $1988 ; 4$ : 52-4.

15. Buus S, Sett A, Colon SM, Miles C, Grey HM. The relation between major histocom patibility complex (MHC) restriction and the capacity of Ia to bind immunogenic peptides. Science 1987 ; 235 : 1353-8.

16. Rock KL, Benacerraf B, Abbas AK. Antigen presentation by hapten-specific B lymphocytes. I. Role of surface immunoglobulin receptors. J Exp Med 1984 ; 160 : 1102-13.

17. Lanzavecchia A. Antigen-specific interac tion between T and B cells. Nature $1985 ; 314$ 537-9.

18. Schutze MP, Leclerc C. Suppression épi topique et vaccins synthétiques. Bull Inst Pasteur 1986 ; 84 : 389-409.

19. Milich DR, McI.achlan A, Thornton GB, Hughes JL. Antibody production to the nucleocapsid and envelope of the hepatitis $B$ virus primed by a single synthetic $\mathrm{T}$ cell site. Nature 1987 ; 329 : 547-9.

20. Leserman L. All in the superfamily : pre sentation of antigen receptors in the contexte of the major histocompatibility complex. Ann Immunol Inst Pasteur (Paris) $1987 ; 138$ : 53-63.

21. Oldstone MBA. Molecular mimicry and autoimmune disease. Cell 1987 ; 50 : 819-20.

22. Urbain J. Is the immune system a functional idiotypic network? Ann Immunol Inst Pasteur (Paris) 1986 ; 137c : 57-100.

23. Coutinho A, Forni L, Holmberg D, Ivars F, Vaz N. From an antigen-centered, clona perspective of immune responses to an organism-centered, network perspective of autonomous activity in a self-referential immune system. Immunol Rev 1984 ; 79 : 151-68.

24. Chalufour A, Bougueleret L, Claverie JM, Kourilsky P. Rare sequence motifs are common constituents of hypervariable antibody regions. Ann Immunol Inst Pasteur (Paris) 1987 ; $138: 671-85$.

25. Singer A, Hathcock KS, Hodes RJ. Sel recognition in allogeneic thymic chimeras. Self recognition by $T$ helper cells from thymus-engrafted nude mice is restricted to the thymic H-2 haplotype. J Exp Med $1982 ; 155$ : 339-44.

26. Singer A, Munitz TI, Gress RE. Specificity of thymic selection and the role of self antigens. Transplant Proc 1987; 19 (6) (sous presse).

27. Maryanski J, Abastado JP, Kourilsky P. Specificity of peptide presentation by a set of hybrid mouse class I MHC molecules. Nature peuvent reconnaître un peptide bien précis présenté par les antigènes du CMH de classe I. Comment ces fibroblastes ou leurs antigènes de classe I «sauraient-ils » discriminer entre les protéines cellulaires et la protéine « étrangère » codée par le gène transfecté ? Il semble beaucoup plus probable que la présentation des peptides dérivés de la protéine étrangere est la trace d'un phénomène plus général, à savoir la présentation, sous forme de peptides, des protéines cellulaires elles-mêmes.

Toute cellule capable de présenter des antigènes étrangers (ce qui est le cas d'un grand nombre de cellules dans l'organisme) serait donc hérissée d'une myriade de peptides du soi. En règle générale, le système immunitaire serait «tolérant » envers ces derniers mais réagirait contre les peptides étrangers apparaissant transitoirement après l'acquisition de la tolérance.

Une généralisation importante touche les cellules du système immunitaire elles-mêmes : les cellules $B$ et $T$ devraient présenter des peptides des immunoglobulines et des récepteurs $T$, que nous avons. baptisés idiopeptides et dont nous avons [1, 3], avec d'autres [7, 8, 20], postulé l'existence. $\mathrm{Si}$ ces idiopeptides existent, on peut imaginer qu'ils jouent un rôle dans les réponses idiotypiques. En fait, il conviendrait tout simplement, dans ces dernières, de distinguer les épitopes B qui comprendraient les idiotopes dans un sens communément accepté (les idiotopes ont été initialement définis comme les épitopes reconnus sur une immunoglobuline par un anticorps monoclonal), et les épitopes $\mathrm{T}$ qui seraient des idiopeptides [l, 3]. L'existence même des réponses idiotypiques conduit à considérer que le système immunitaire n'est pas tolérant vis-à-vis d'une partie au moins des idiopeptides. Ceci nous a conduit à distinguer le « soi somatique » du «soi immunologique ». Ce dernier est composé de l'ensemble des idiopeptides non «tolérisés » présentés au système immunitaire. Le «soi somatique » recouvre l'ensemble des peptides des protéines du soi (hormis les idiopeptides) présentés par les molécules du $\mathrm{CMH}$ (principalement des cellules somatiques).

Le «sol», au sens immunologique du terme, est donc, dans cette perspective, défini comme un ensemble de peptides dont nous avons indiqué plus haut qu'il est sélectionné par les antigènes du CMH de l'individu. En d'autres termes, des individus par ailleurs identiques mais portant des haplotypes du $\mathrm{CMH}$ distincts, doivent présenter des ensembles de peptides différents, de sorte que leur «soi » au sens immunologique, sera différent. Cette proposition doit être nuancée. Outre les antigènes classiques polymorphes, il existe des antigènes du CMH dits non classiques et peu polymorphes (antigènes $\mathrm{Qa}$, TL, 37 chez la souris) et d'autres antigènes associés à la $\beta$-2-microglobuline non liés au CMH (antigènes $\mathrm{CDl}$ chez l'homme). Si ces antigènes ont une fonction de présentation - ce qui n'est qu'une hypothèse - ils pourraient presenter au système immunitaire des jeux de peptides (ou d'idiopeptides) invariants d'un individu à l'autre.

\section{Quelques corrélats du modèle du « soi peptidique »}

Le modèle du «soi peptidique» permet de formuler un certain nombre d'hypothèses secondaires et de prédictions qui peuvent être soumises à l'expérience [1-3].

Les antigènes mineurs d'histocompatibilité. Si les peptides du soi sont présentés, il se peut que certains des polymorphismes qui touchent des protéines (souvent domestiques) de l'organisme, se traduisent par la présentation de peptides variants a la surface des greffons, provoquant leur rejet. Certains antigènes mineurs d'histocompatibilité pourraient donc être des peptides présentés par les antigènes du CMH. Si tel est le cas, cela pourrait expliquer pourquoi leur purification biochimique a le plus souvent échoué. 
Les antigènes tumoraux. Le même argument pourrait valoir pour certains antigènes tumoraux qui, dans cette perspective, seraient des peptides dérivés de protéines du soi. L'un des corrélats du modèle du « soi peptidique » est d'élargir la notion de surveillance immunitaire aux protéines intracellulaires, et ceci sous deux formes. D'une part, certaines mutations dans ces dernières pourront être signalées au système immunitaire. D'autre part, un argument quantitatif veut qu'une protéine cellulaire très peu abondante soit considérée comme «étrangère », en ce sens que la compétition interne pour la présentation des protéines du soi et l'existence d'un seuil minimal de détection l'élimine de la scène immunologique. Ce pourrait être, entre autres, le cas de protéines mineures, par exemple régulatrices qui, surexprimées dans certaines tumeurs, pourraient ainsi se signaler au système immunitaire.

Les maladies auto-immunes. Un raisonnement similaire peut s'appliquer à certaines maladies autoimmunes. Un dérèglement physiologique acquis entraînant la présentation de protéines rares (et donc « étrangères ») déclencherait une attaque immunitaire contre les tissus ou les cellules, sièges de ce dysfonctionnement. Une autre source de dérèglement pourrait provenir de coïncidences accidentelles, dans le contexte de certains haplotypes du $\mathrm{CMH}$, entre des peptides du soi et des peptides provenant d'agents infectieux [21] ou, peut-être, d'idiopeptides. Les réactions immunitaires dirigées contre des cellules présentant les derniers se trouveraient, de ce fait, dirigées également contre des cellules de l'organisme.

L'existence possible d'un réseau «idiopeptidique». L'existence postulée d'éléments régulateurs additionnels dans le système immunitaire, c'est-à-dire les idiopeptides, est riche d'implications potentielles. Dans la théorie du réseau idiotypique de Jerne [22, 23], des anticorps sont synthétisés en cascade $(\mathrm{Abl} \rightarrow \mathrm{Ab} 2 \rightarrow \mathrm{Ab} 3)$
(Ab2 étant un anticorps dirigé contre $\mathrm{Abl}$, et $\mathrm{Ab} 3$ dirigé contre $\mathrm{Ab} 2$; certains des Ab3 peuvent donc ressembler à des $\mathrm{Abl}$ ). Dans le réseau «idiopeptidique» tel que nous l'avons postulé, il existe une boucle régulatrice telle que Abl stimule la synthèse de Abl [23], sans qu'il y ait nécessairement relais par un Ab2. Ceci est dû au fait que la cellule B qui expose Abl à sa surface expose aussi des idiopeptides $\mathrm{Abl}$ dérivés de sa chaîne lourde et de sa chaîne légère. Cette cellule peut donc être directement activée par des cellules $T$ auxiliaires «idiopeptidiques » activées par les $\mathrm{Abl}$ euxmêmes. Cette particularité, et la dissociation des Abl en idiopeptides dérivés des chaînes lourdes et légères confèrent au réseau idiopeptidique des propriétés logiques différentes de celles du réseau de Jerne [3] dont le caractère fonctionnel n'est pas établi, même si des cascades d'anticorps (Abl, Ab2, Ab3) ont été observées dans des immunisations successives, et parfois chez le même animal. Les propriétés homéostatiques de ces boucles de régulation (qui peuvent faire intervenir des signaux négatifs et donc des cellules suppressives) sont importantes à considérer parce qu'elles pourraient jouer un rôle clé dans la mémoire immunitaire [3].

\section{L'univers des séquences peptidiques}

Si les prémisses du modèle du «soi peptidique» sont correctes, un épitope $T$ dérivé d'une protéine immunogène doit posséder une séquence distincte de celles des protéines du soi somatique. Les épitopes $T$ dérivés de ces dernières ont, en effet, dû faire l'objet d'une «tolérisation», quel que soit le mécanisme de celle-ci. Ceci a conduit à analyser les séquences de peptides dont il est expérimentalement démontré qu'ils possèdent une immunogénicité au niveau des cellules $\mathrm{T}$. La comparaison des séquences avec celles du soi somatique suggère qu'effectivement les peptides immunogènes $T$ diffèrent en séquence des protéines du soi d'une façon qui peut être appréhendée par l'algorithme utilisé ([1] et Claverie et al., en préparation). L'intérêt de cette observation est qu'à l'inverse elle pourrait fournir un critère de prédiction des épitopes T. De la même manière, nous avons comparé les séquences de nombreuses chaînes lourdes et légères d'immunoglobulines à celles des protéines somatiques [24]. Il semblerait que certaines des régions hypervariables soient constituées de séquences «étrangères au soi somatique», ce qui serait conforme aux hypothèses de départ. L'univers des séquences peptidiques comprendrait donc plusieurs sous-ensembles: tout d'abord le sous-ensemble des séquences des protéines somatiques dont un sous-sous-ensemble est sélectionné par les antigènes du CMH ; puis le sous-ensemble des séquences idiopeptidiques, et enfin celui des séquences du monde extérieur. Ces propositions, toutefois, doivent être traitées avec la plus grande prudence. Elles ne reposent, à ce jour, que sur des corrélations qui, bien que statistiquement significatives, n'ont pas valeur de preuve.

\section{Le soi est-il uniquement peptidique?}

Selon le modèle du soi peptidique (figure 2, page 182), le soi, au sens immunologique du terme, est constitué par l'ensemble des peptides des protéines somatiques qui sont sélectionnés et présentés par les antigènes du CMH. Si l'on change d'haplotype, on change le jeu des peptides du soi [2]. Ce faisant, on crée des opportunités pour des réactions croisées, de sorte que l'on comprend bien pourquoi un récepteur $T$, très spécifique dans un haplotype donné, présente souvent des réactions croisées dans un autre haplotype; ou encore pourquoi l'immunisation d'un animal avec des cellules d'un autre haplotype produit des réactions alloréactives a une fréquence très élevée. Dans les deux cas, les myriades de peptides du soi différents selon l'ha- 


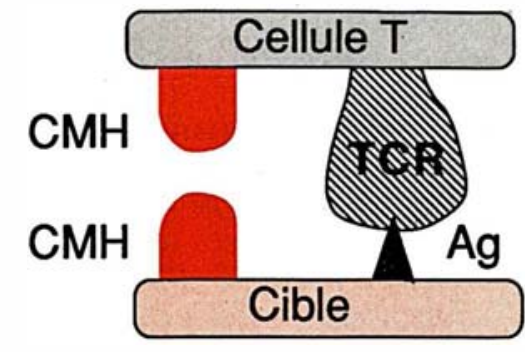

modèle d'intimité
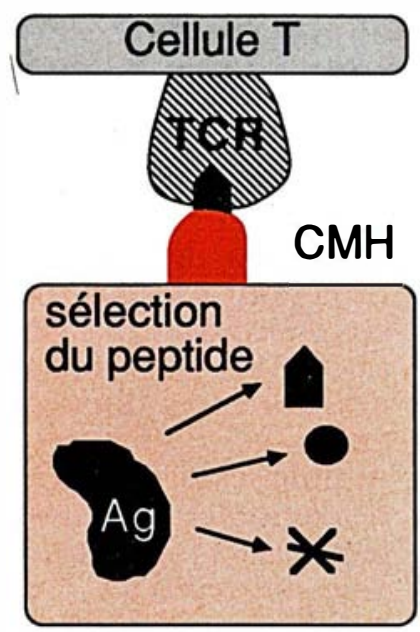

1

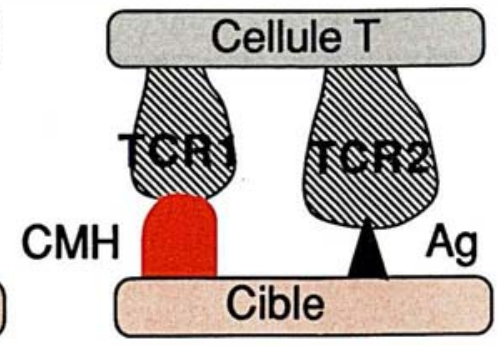

modèle à 2 récepteurs

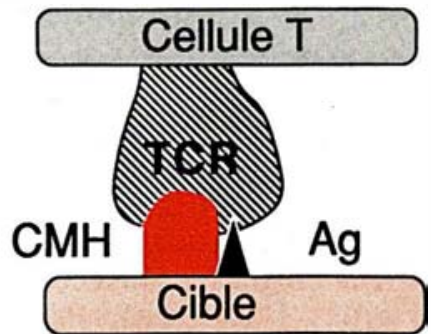

modèle du soi altéré
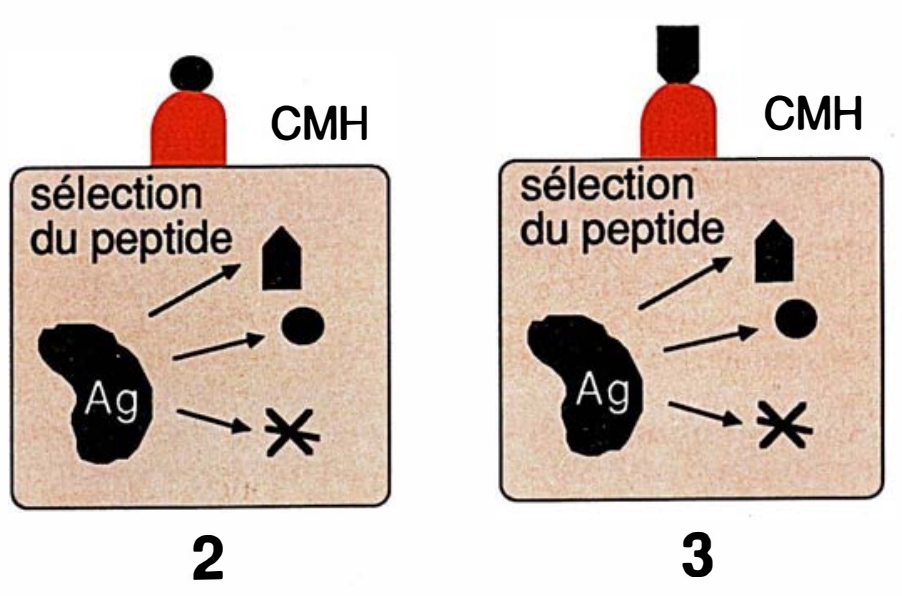

Modèle du soi peptidique

Figure 2. Le phénomène de la restriction CMH : la version extrême du modèle du soi peptidique par rapport aux modèles plus anciens. Alors que les modèles anciens (modèle d'intimité, modèle à 2 récepteurs, modèle du soi altéré) incorporent la notion de reconnaissance de l'«antigène „ du CMH (complexe majeur d'histocompatibilité), ou de sa coreconnaissance avec l'antigène nominal par le lymphocyte T, le modele du soi peptidique renverse la perspective. Les molécules du CMH (complexe majeur d'histocompatibilité) passent du statut d'antigène à celui de récepteur de peptides. L'expression d'haplotypes différents du CMH dans deux hôtes syngéniques entraînent la sélection et la présentation d'un ensemble de peptides non identiques après la digestion des mêmes protéines du soi. (1) L'antigène est dégradé et ses peptides sont présentés. (2) Un autre peptide dérivé de l'antigène est présenté. (3) Le même peptide est présenté différemment. Ce serait une des bases du phénomène d'alloréactivité. Le schéma présenté reflète la version extrême, provocatrice (hypothèse b dans le texte) de la reconnaissance des seuls peptides (et, peut-être, de résidus non polymorphes de l'antigène du CMH) par le récepteur $T$. Dans une version moins stricte certains résidus polymorphes pourraient interagir avec certains récepteurs $T$, mais de façon non systématique, au hasard des micro-clones de cellules Tqui se développent dans l'organisme. Ag = antigène; $T C R=T$-cell receptor (récepteur pour l'antigène des lymphocytes T). (Nous remercions J.P. Abastado ne nous avoir permis de reproduire cette figure.)

plotype sont source de réaction [2, 9] (figure 2).

Peut-on, dès lors, réduire le « soi » aux peptides sélectionnés et présentés par les molécules du $\mathrm{CMH}$ ? Ou faut-il inclure dans le «soi » les molécules du CMH 182
$\mathrm{CMH}$, souvent appelés antigènes du soi, font-ils vraiment partie du soi, ou servent-ils seulement à spécifier, en le sélectionnant, le soi ? La traduction moléculaire de ces interrogations est celle-ci : le récepteur des cellules $T$ reconnaît-il (a) les peptides présentés par les antigènes du $\mathrm{CMH}$ ainsi que tous les résidus polymorphes de ces derniers? ou bien (b) seulement les peptides présentés $[2,9]$ (et, peut-être, des résidus non polymorphes des antigènes du CHM) (figure 2)? ou bien (c) les peptides présentés et, de façon 
spécifique et constante, quelques résidus polymorphes des antigènes du $\mathrm{CMH}$ ? ou, enfin (d) les peptides présentés et, de manière contingente, certains des résidus polymorphes des molécules du $\mathrm{CMH}$ ?.

Ces questions ne sont pas un simple exercice sémantique. Elles renvoient en effet au problème épineux de l'«apprentissage » du soi par le système immunitaire et du développement du répertoire $T$ au cours de l'ontogénie. Les phénomènes de restriction $\mathrm{H}$-2 (chez la souris) et HLA (chez l'homme) ont été, peu après leur découverte, interprétés comme signifiant une co-reconnaissance obligée des antigènes présentés et des moléculesdu CMH présentatrices (d'où divers modèles illustrés dans la figure 2). Cette idée a été renforcée par les expériences d'éducation thymique qui suggèrent elles aussi un «apprentissage » de la reconnaissance des antigènes du $\mathrm{CMH}$ dans le thymus [25]. Ces interprétations posent de sévères contraintes sur le récepteur T qui doit reconnaître très spécifiquement les antigènes du CMH du soi ainsi que tout l'inconnu du monde extérieur. Ceci force à construire des hypothèses qui, d'une manière ou d'une autre, introduisent une relation de similitude entre le soi et le non soi. Par exemple, selon Singer et al. [26], au cours de l'ontogénie du système immunitaire, les cellules $T$ qui reconnaissent très spécifiquement les antigènes du soi, présentés par les antigènes du $\mathrm{CMH}$, seraient inactivées ou détruites. En revanche, celles qui les reconnaîtraient faiblement seraient stimulées, de sorte que la reconnaissance du non soi coïnciderait avec la reconnaissance «floue» du soi.

L'alternative offerte par le modèle du soi peptidique lève cette difficulté conceptuelle. Dans sa forme extrême [2] (hypothèse (b) ci-dessus), le récepteur $T$ pourrait ne pas reconnaître les résidus polymorphes des antigènes du CMH. Dans une forme moins brutale (d), le récepteur $T$ pourrait entrer en contact, mais de façon non systématique, avec des résidus poly-

$\mathrm{m} / \mathrm{s} \mathrm{n}^{\circ} 3$ vol. 4 , mars 88 morphes des antigènes du $\mathrm{CMH}$, tantôt certains, tantôt d'autres, parfois aucun, au hasard des micro-clones qui se développent au cours de l'ontogénie. A notre connaissance, aucune expérience à ce jour ne permet de trancher clairement entre les différentes hypothèses [27]. L'une semble toutefois hautement improbable: c'est l'hypothèse (a) où tous les résidus polymorphes des antigènes du CMH sont reconnus par le récepteur $\mathrm{T}$. La structure tridimensionnelle de l'antigène HLAA2 indique en effet que certains de ces résidus sont enfouis dans la protéine et quasiment inacessibles $[13,14]$. Enfin, on notera que l'hypothèse (b) ne rend pas facilement compte des expériences d'éducation thymique, alors que l'hypothèse (d) n'est pas incompatible avec ces dernières.

\section{Perspectives}

Les perspectives d'expérimentation offertes par le modèle du «soi peptidique» sont nombreuses et touchent à plusieurs domaines importants dont celui de la manipulation des réponses immunitaires en général et des vaccinations en particulier. Si l'existence des idiopeptides était démontrée, ceux-ci pourraient constituer une nouvelle classe d'agents pharmacologiques. L'élucidation des règles qui contrôlent l'interaction entre peptides présentés et antigènes du $\mathrm{CMH}$ permettront de mieux utiliser des épitopes $T$ régulateurs, dans une logique des couplages qui a été brièvement évoquée plus haut. La vertu d'un modèle réside sans doute moins dans son exactitude que dans sa capacité à organiser les faits et à stimuler sa vérification ou son invalidation par l'expérience. Le modèle du soi peptidique permet d'expliquer de façon simple nombre de résultats immunologiques connus, et de formuler diverses hypothèses de travail qui sont en cours d'expérimentation. C'est en ce sens, nous l'espérons, que ce modèle trouvera son utilité

\section{Summary}

Recent developments in immunology have emphasized the fundamental distinction between $B$ (recognized by antibodies) and $\mathrm{T}$ epitopes (recognized by $T$ cell receptors). In general, T epitopes appear to be peptides derived from processed antigens and selectively presented by class I and class II molecules of the major histocompatibility complex (MHC). The latter can, therefore, be viewed as peptide receptors endowed with a certain specificity. The concept that selected peptides derived from self proteins are permanently presented by class I and class II MHC molecules is the basis of the peptidic self model. Its numerous implications include: (a) a definition of the self (versus non-self), as the set of peptides derived from self proteins and selected by the MHC molecules of the individual; (b) the prediction of the existence of the so-called «idiopeptides » which possibly constitute a new class of regulatory elements in the immune system, suggesting an «idiopeptidic» network; (c) working hypotheses on the molecular nature of certain minor histocompatibility antigens and tumor specific antigens, as well as on the origin of certain auto-immune diseases.

\section{TIRÉS A PART}

Ph. Kourilsky : unité de biologie moléculaire du gène, Inserm U. 277, Institut Pasteur, 25, rue du Docteur-Roux, 75724 Paris Cedex 15, France. 